\title{
Effects of stenting on blood flow in a coronary artery network model
}

doi:10.1533/abbi.2005.0023

\author{
R. Raghu, A. Pullan and N. Smith \\ Department of Engineering Science and Bioengineering Institute, The University of Auckland, Private Bag 92019, \\ Auckland, New Zealand
}

\begin{abstract}
The effect of stenting on blood flow is investigated using a model of the coronary artery network. The parameters in a generic non-linear pressure-radius relationship are varied in the stented region to model the increase in stiffness of the vessel due to the presence of the stent. A computationally efficient form of the Navier-Stokes equation is solved using a Lax-Wendroff finite difference method. Pressure, vessel radius and flow velocity are computed along the vessel segments. Results show negative pressure gradients at the ends of the stent and increased velocity through the middle of the stented region. Changes in local flow patterns and vessel wall stresses due to the presence of the stent have been shown to be important in restenosis of vessels. Local and global pressure gradients affect local flow patterns and vessel wall stresses, and therefore may be an important factor associated with restenosis. The model presented in this study can be easily extended to solve flows for stented vessels in a full, anatomically realistic coronary network. The framework to allow for the effects of the deformation of the myocardium on the coronary network is also in place.
\end{abstract}

Key words: Coronary stent, Lax-Wendroff method, coronary artery model, Navier-Stokes equations.

\section{INTRODUCTION}

Stents are metallic mesh tubes that are inserted into arteries to keep them open. A stent is generally placed in an occluded artery (such as coronary arteries) following a balloon angioplasty (also known as percutaneous transluminal coronary angioplasty). Balloon angioplasty involves inserting a balloon catheter into the femoral artery and guiding it to the blockage site in the coronary artery. The balloon is then inflated to widen the artery and remove the blockage. The catheter is removed, and then the stent catheter is introduced into the system. The stent is placed over a deflated balloon on the catheter. Once the stent is at the blockage site, the balloon is inflated and the stent is deployed to keep the artery open.

Stenting can reduce acute complications of angioplasty as well as the restenosis rate (Jowett and Thompson 2003). With just balloon angioplasty (and no stent implantation), up to $50 \%$ of cases develop restenosis. This restenosis is

Corresponding Author:

Dr A. Pullan

Department of Engineering Science and Bioengineering Institute

The University of Auckland, Private Bag 92019

Auckland, New Zealand

Tel: +649-373-7599; Fax: +649-367-7157

Email: a.pullan@auckland.ac.nz due to arterial remodelling (shrinkage) and neointimal hyperplasia (Jowett and Thompson 2003). Restenosis rates are lower with stenting than without because of better initial patency of the artery. However, restenosis still occurs in about $20 \%$ to $30 \%$ of cases mainly due to neointimal proliferation. Stenting is also said to improve the safety and efficiency of balloon angioplasty procedures since the need for emergency coronary artery bypass graft surgery is reduced (Jowett and Thompson 2003; Yock et al. 2003).

Some of the stenosis locations that are targeted for stent implantation (and used in many modelling studies) are in the left anterior descending (LAD), right coronary and circumflex arteries (Wentzel et al. 2000; Capozzolo et al. 2001; Hsieh et al. 2001; LaDisa et al. 2003; Zhu et al. 2003).

Stents can cause longitudinal straightening of the vessels due to their stiffness and can also cause the enlargement of the lumen of the vessels due to their radial force ( $\mathrm{Zhu}$ et al. 2003; Tortoriello and Pedrizzetti 2004). The presence of the stent introduces a compliance mismatch with the surrounding portion of the vessel. Several studies have modelled the biomechanics of stents including initial stent expansion and deformation (Barragan et al. 2000; Etave et al. 2001; Tan et al. 2001; Migliavacca et al. 2002). Other studies have presented experimental results and models of flow and flow-tissue interactions in the local stented region of arteries (Rolland et al. 1999; Berry et al. 2000; Moore and Berry 2002; Benard et al. 2003; LaDisa et al. 2003; 


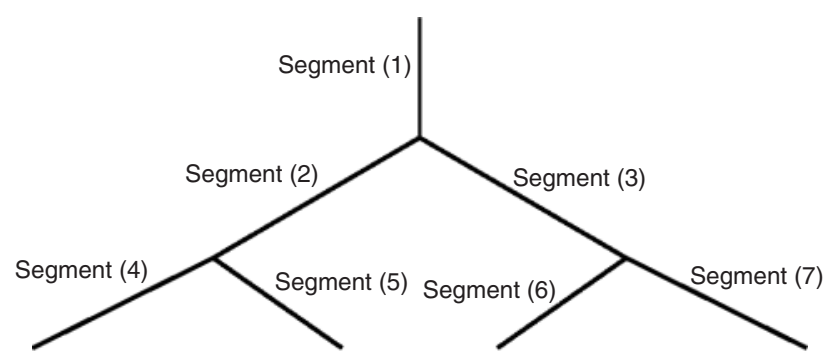

(a)

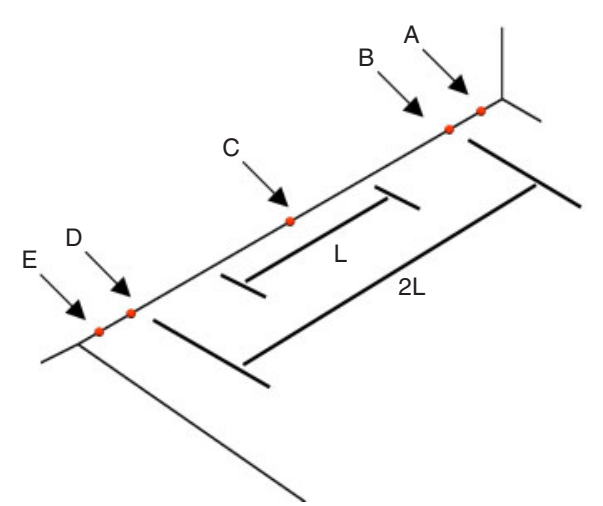

(b)

Figure 1 (a) Coronary artery network model—labelled in the figure are the numbers of the network vessel segments. The lengths of the segment are: (1) is $20 \mathrm{~mm},(2)$ and (3) are $40 \mathrm{~mm},(4)$ and (7) are $34 \mathrm{~mm},(5)$ and (6) are $26.5 \mathrm{~mm}$. (b) Close-up of segment 2 where the stent was placed- $L$ is the length of the stent in place ( $15 \mathrm{~mm}$ in this study), $2 L$ denotes the region where the stent affects the material properties of the vessel, $\mathrm{A}-\mathrm{E}$ are points along the vessel segment at which results are presented later in the article.

Tortoriello and Pedrizzetti 2004). Many such studies are mainly concerned with the local changes in the flow and the implications on restenoses of stented arteries.

One-dimensional blood flow models that assume a radial velocity profile are increasingly being developed in the literature (Smith et al. 2002; Formaggia et al. 2003; Sherwin et al. 2003). The simplifications in these models remove explicit calculation of local effects such as circumferential stress. However, the formulation is computationally efficient, and thus provides the ability to simulate blood flow in large networks. To our knowledge, this is the first study to investigate computationally the effects of stenting on the flow in a network.

In this article, we present such a computationally efficient formulation to study the effects of stenting an artery segment on the local, upstream and downstream flow pressures, velocities and vessel radii. The principles of modelling blood flow used in this study can be extended in a straightforward manner to anatomically realistic coronary artery meshes. The framework to allow the mechanics of the heart is already in place for more detailed future models (Smith et al. 2005). A finite difference grid based on an underlying finite element coronary artery mesh is used in this study (Figure 1).

This study presents a one-dimensional model of coronary artery blood flow in the unstented and stented cases for an assumed pressure pulse. In the stented case, the compliance mismatch is modelled by changing the form of the pressure-radius relationship within the stiffer stented region compared with the surrounding vessels. The resulting transient and steady-state pressures, velocities and vessel radii are compared with each other and physiological conclusions are drawn from the results.

\section{MODEL DESCRIPTION}

\section{Blood flow equations}

\section{Single vessel}

The blood flow model used in this study is the one presented by Smith et al. (2002). Blood is modelled as an incompressible, homogeneous, Newtonian fluid. The Navier-Stokes equations govern the Newtonian fluid flow. A cylindrical coordinate system $(r, \theta$ and $x)$ is used in the model, with $x$ representing the local vessel axial direction. Assuming that the velocity in the circumferential $(\theta)$ direction is zero and following the derivation given in Smith et al. (2002), the Navier-Stokes equations that govern the Newtonian fluid flow reduce to (1).

$$
\begin{aligned}
& \frac{\partial V}{\partial t}+(2 \alpha-1) V \frac{\partial V}{\partial x}+2(\alpha-1) \frac{V^{2}}{R} \frac{\partial R}{\partial x}+\frac{1}{\rho} \frac{\partial p}{\partial x} \\
& \quad=-2 \frac{\nu \alpha}{\alpha-1} \frac{V}{R^{2}}
\end{aligned}
$$

where $V$ is the average velocity, $p$ is the pressure, $R$ is the inner vessel radius, $\rho$ is the fluid density, $v$ is the fluid viscosity and $t$ is the time. The term $\alpha$ is a nondimenionalised parameter, which defines the radial velocity profile that can vary between 1.33 , fully developed flow, and 1.0, corresponding to a flat plug flow profile (for more details, see Smith et al. 2002).

The equation of conservation of mass is given by (2).

$$
\frac{\partial R}{\partial t}+V \frac{\partial R}{\partial x}+\frac{R}{2} \frac{\partial V}{\partial x}=0 .
$$

To solve the system, another equation describing the relationship between pressure and inner vessel radius is needed. The vessel wall is assumed to be elastic and any viscoelastic effects are ignored in this model. An empirical relationship shown in (3) is established between the pressure and radius.

$$
p(R)=G_{0}\left[\left(\frac{R}{R_{0}}\right)^{\beta}-1\right],
$$

where $R_{0}$ is the unstressed vessel radius and $G_{0}$ and $\beta$ are parameters that define wall behaviour. A two-step LaxWendroff finite difference technique is used to solve the above equations. 


\section{Bifurcation model}

A bifurcation model is necessary to model flow through the branches in the artery network. The bifurcation model used in this study was presented by Smith et al. (2002). In the model, the junction of three tubes $(a, b$ and $c)$ is approximated as the elastic tubes short enough for velocity along them to be assumed constant and for losses due to fluid viscosity to be negligible. It is also assumed that no fluid is stored within the junction. Let $a_{1}$ be the finite difference grid point at the end of vessel a (the parent vessel) entering the junction and let $a_{2}$ be the point proximal to it. Let $b_{1}$ and $c_{1}$ be the grid points at the beginning of the daughter vessels ( $b$ and $c)$ and let the distal points be denoted $b_{2}$ and $c_{2}$, respectively.

The conservation of mass at any given time through the junction is then governed by (4), where $F_{a_{1}}, F_{b_{1}}$ and $F_{c_{1}}$ are the flows through each junction segment and $p_{0}$ is the pressure at the junction centre.

$$
F_{a_{1}}-F_{b_{1}}-F_{c_{1}}=0 \text {. }
$$

In a segment of length $l_{\mathrm{a}}$ and radius $R_{\mathrm{a}}$ of tube a, the conservation of momentum of fluid is given by (5).

$$
\pi R_{\mathrm{a}}^{2}\left(p_{\mathrm{a}}-p_{0}\right)=\frac{\partial\left(\rho l_{\mathrm{a}} \pi R_{\mathrm{a}}^{2} V_{\mathrm{a}}\right)}{\partial t} .
$$

Similarly, the conservation of momentum equations can be written for tubes $b$ and $c$. These are then expanded using a central difference representation about the $\left(k+\frac{1}{2}\right)$ time step.

\section{Arterial properties}

For all simulations, blood density $\rho$, viscosity $\mu$, and the flow profile parameter $\alpha$ have been set to $1.05 \mathrm{e}-03 \mathrm{~g} \mathrm{~mm}^{-3}$, $3.2 \mathrm{~mm}^{2} \mathrm{~s}$ and 1.1, respectively. The difference between unstented and stented vessels lies in the pressure-radius relationship defined for each case (Equation (3)), in particular, the descriptions of $G_{0}$ and $\beta$ used in each case.

\section{Unstented vessel}

For an unstented vessel, $G_{0}$ and $\beta$ values are taken to be constant along the entire vessel. The values used in this model are $G_{0}=G_{0 \mathrm{C}}=10.0 \mathrm{kPa}$ and $\beta=2.0$. These chosen values for the constants are typical of values fitted from the experimental data of Carmines et al. (1991).

\section{Stented vessel}

Because of the presence of the stent, the vessel becomes stiff. Therefore, the pressure-radius relationship within the stented region will be different from the one outside the stented region. As the effect of the stent in the network is modelled by altering the mechanical properties of the vessel wall, the shape of the stent is assumed to implicitly conform to the shape of the vessel wall and not substantially alter the haemodynamic characteristics, that is, cylindrical, axi-symmetric flow. Thus, specifics such as stent geometry and strut size are beyond the scope of inclusion in network models via this technique.
Since the vessel is a continuous structure, this change in the pressure-radius relationship between the unstented and stented regions is assumed to occur in a smooth manner without discontinuities. This change in the pressureradius relationship is described by the gradual change in $G_{0}$ and $\beta$ values along the length of the vessel, where the stent is assumed to have an effect. The total length of the region where the stent is assumed to have an effect is taken to be twice the length of the stent. The variation in $G_{0}$ and $\beta$ values is given by the relationships shown in (6) and (7), which are adapted from the work of Tortoriello and Pedrizzetti (2004).

$$
\begin{aligned}
& G_{0}= \begin{cases}G_{0 \mathrm{C}} & x<x_{\mathrm{s}}, x>\left(x_{\mathrm{s}}+2 L\right) \\
G_{0 \mathrm{C}}\left(\frac{E h(x)}{(\bar{R}(x))^{2}}\right) & x_{\mathrm{s}} \leq x \leq\left(x_{\mathrm{s}}+2 L\right)\end{cases} \\
& \beta= \begin{cases}\beta_{\mathrm{C}} & x<x_{\mathrm{s}}, x>\left(x_{\mathrm{s}}+2 L\right) \\
\beta_{\mathrm{C}}\left(1+\Delta \beta \mathrm{e}^{\left.-\left(2\left(\frac{x-x_{\mathrm{s}}}{L}\right)-2\right)^{n}\right)}\right. & x_{\mathrm{s}} \leq x \leq\left(x_{\mathrm{s}}+2 L\right)\end{cases}
\end{aligned}
$$

In (6) and (7), $x$ is the axial distance from the start of a vessel segment, $x_{\mathrm{S}}$ is the distance at which the effect of the stent is assumed to start, $L$ is the length of the stent, $G_{0 \mathrm{C}}$ and $\beta_{\mathrm{C}}$ are the constant parameters as used in the unstented vessel and $n$ is used to control the steepness of the transition of the $G_{0}$ and $\beta$ values from the unstented to the stented regions and back. The $x$-dependent functions $E h(x)$ and $\bar{R}(x)$ are given by (8) and (9).

$$
\begin{aligned}
E h(x) & =1+\Delta E h \mathrm{e}^{-\left(2\left(\frac{x-x_{\mathrm{s}}}{L}\right)-2\right)^{n}} . \\
\bar{R}(x) & =1+\Delta \bar{R} \mathrm{e}^{-\left(2\left(\frac{x-x_{\mathrm{s}}}{L}\right)-2\right)^{n}} .
\end{aligned}
$$

For this model, the constants $\Delta E h$ and $\Delta \bar{R}$ were taken to be 60 and 0 , respectively, thus making $\bar{R}(x)=1$. The function $\bar{R}(x)$ can actually be used to model any increase in unstressed vessel radius due to the expansion of the stent. For this model, however, the unstressed vessel radius was taken as constant in the stented and unstented regions. According to the experimental measurements of pressure and vessel diameter conducted by Rolland et al. (1999), the effects of the stent can be felt up to $10 \mathrm{~mm}$ on either side of the stent. Therefore, the value of $n$ was taken as 4 , giving a less steep transition for the $G_{0}$ and $\beta$ values compared with that reported in Tortoriello and Pedrizzetti (2004), where a value of 8 was assigned to $n$. Figure 2 shows an example of the variation of $G_{0}$ and $\beta$ along a stented vessel.

\section{Steady-state solution}

\section{Stented vessel}

Here, we derive a steady-state solution for the stented vessel case as a means of verifying the numerical solution procedure. By setting all time-dependent terms in (1) to be zero, and using vessel area $S=\pi R^{2}$ and a constant flow rate $Q=V S$, from the conservation of mass principle, we obtain (10).

$$
-\frac{\alpha Q^{2}}{(S(x))^{3}} \frac{\mathrm{d} S}{\mathrm{~d} x}+\frac{1}{\rho} \frac{\mathrm{d} p}{\mathrm{~d} x}=-2 \pi \frac{v \alpha}{\alpha-1} \frac{Q}{(S(x))^{2}} .
$$




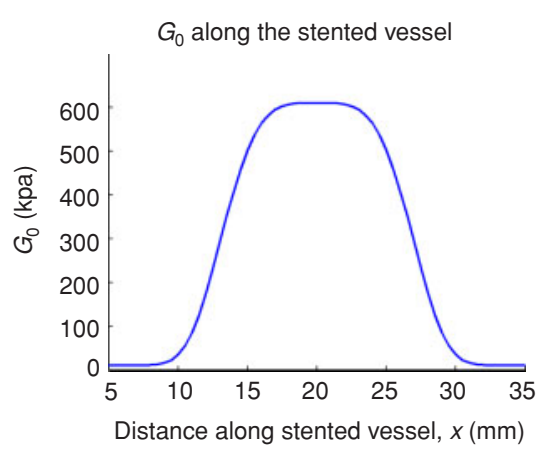

(a)

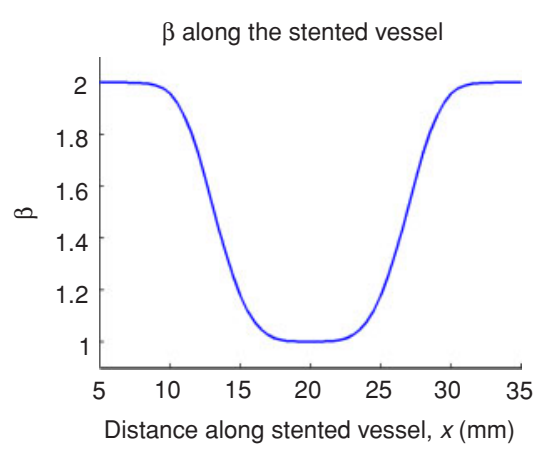

(b)

Figure 2 (a) $G_{0}$ and (b) $\beta$ values computed from (6) and (7) along a stented vessel with $x_{\mathrm{s}}=5 \mathrm{~mm}$, stent length $L=15 \mathrm{~mm}$.

Substituting vessel area into (3), we obtain (11),

$$
p(S)=G_{0}\left[\left(\frac{S}{S_{0}}\right)^{\beta / 2}-1\right]=G_{0}(x) H(x),
$$

where $G_{0}(x)$ is given by the appropriate part of (6) and

$$
H(x)=\left(\frac{S(x)}{S_{0}}\right)^{\beta(x) / 2}-1 .
$$

Equations (11) and (12) can be used to give an expression for $\frac{\mathrm{d} p}{\mathrm{~d} x}$ (Equation (13)) in the region where $x_{\mathrm{s}} \leq x \leq\left(x_{\mathrm{s}}+\right.$ $2 L)$. Note that in the stented region of the vessel both $G_{0}$ and $\beta$ are functions of the axial distance $x$.

$$
\frac{1}{\rho} \frac{\mathrm{d} p}{\mathrm{~d} x}=\frac{1}{\rho}\left[\frac{\mathrm{d} G_{0}}{\mathrm{~d} x} H(x)+G_{0}(x) \frac{\mathrm{d} H}{\mathrm{~d} x}\right] .
$$

The derivative terms in (13) are given by (14) and (15).

$$
\begin{aligned}
\frac{\mathrm{d} G_{0}}{\mathrm{~d} x} & =G_{0 \mathrm{C}}\left[\frac{\frac{\mathrm{d}(E h)}{\mathrm{d} x}(\bar{R}(x))^{2}-2 \bar{R}(x) E h(x) \frac{\mathrm{d}(\bar{R}(x))}{\mathrm{d} x}}{(\bar{R}(x))^{4}}\right] . \\
\frac{\mathrm{d} H}{\mathrm{~d} x} & =\frac{1}{2}[H(x)+1]\left[\frac{\mathrm{d} \beta}{\mathrm{d} x} \ln \left(\frac{S(x)}{S_{0}}\right)+\frac{\beta(x)}{S(x)} \frac{\mathrm{d} S}{\mathrm{~d} x}\right] .
\end{aligned}
$$

Equations (7)-(9) can be easily differentiated to give $\frac{\mathrm{d} \beta}{\mathrm{d} x}$, $\frac{\mathrm{d}(E h)}{\mathrm{d} x}$ and $\frac{\mathrm{d}(\bar{R})}{\mathrm{d} x}$. So substituting (14) and (15) into (13), we have an expression for $\frac{\mathrm{d} p}{\mathrm{~d} x}$ in terms of $x$ and $S$ such that it can be used in (10). This gives us an ordinary differential equation (ODE) describing $\frac{\mathrm{d} S}{\mathrm{~d} x}$ as shown in (16). This differential equation cannot be solved analytically. It is solved numerically using the MATLAB software package with a Runge-Kutta method based on the work of Dormand and Prince (1980). The vessel area $S(x)$ from this steady-state solution is later compared with the flow simulation results to verify the simulations described below (Figure 6).

$$
\left[-\frac{\alpha Q^{2}}{(S(x))^{3}}+\frac{1}{2 \rho} G_{0}(x)(H(x)+1) \frac{\beta(x)}{S(x)}\right] \frac{\mathrm{d} S}{\mathrm{~d} x}
$$

$$
\begin{aligned}
= & -2 \pi \frac{v \alpha}{\alpha-1} \frac{Q}{(S(x))^{2}}-\frac{1}{\rho} \frac{\mathrm{d} G_{0}}{\mathrm{~d} x} H(x) \\
& -\frac{1}{2 \rho} G_{0}(x)(H(x)+1) \frac{\mathrm{d} \beta}{\mathrm{d} x} \ln \left(\frac{S(x)}{S_{0}}\right) .
\end{aligned}
$$

\section{FLOW SIMULATIONS}

Pressure boundary conditions were set for the inlet at the top of vessel segment 1 and at the outlets at the bottom of segments 4, 5, 6 and 7. Exit pressures were held at $2 \mathrm{kPa}$ for the duration of the flow simulations. This pressure was set to the lower end of the range reported by Defily et al. (1993) for a small epicardial vessel of the same size.

Simulations were carried out for two different cases of inlet pressure under unstented and stented conditions: (I) linear increase in inlet pressure from $2 \mathrm{kPa}$ to $3 \mathrm{kPa}$ over $0.25 \mathrm{~s}$ and inlet pressure held at $3 \mathrm{kPa}$ up to $1.0 \mathrm{~s}$ and (II) a single sinusoidal pressure pulse squared followed by a constant pressure value held at $2 \mathrm{kPa}$ as given by (17).

$$
p_{\mathrm{i}}=\sin ^{2}\left(\pi \frac{t}{t_{f}}\right)+2
$$

where $p_{\mathrm{i}}$ is the inlet pressure and $t_{f}$ is the period of the curve ( $0.25 \mathrm{~s}$ in this case). The stent was assumed to have a length $L$ of $15 \mathrm{~mm}$ with an expanded radius $R_{0}$ of $1.5 \mathrm{~mm}$. The unstressed vessel radii for all segments of the network were also taken to be $1.5 \mathrm{~mm}$.

All simulations were carried out on a High Performance Computer maintained at The University of Auckland. The machine (Silicon Graphics Origin 3400 model) contains 16 processors (MIPS designed, $500 \mathrm{MHz}, \mathrm{R} 14000$ ) with 16 GB physical DRAM running the Silicon Graphics IRIX 6.5.13 operating system — only one processor in the system was used for running the simulations. The software used for the simulations was CMISS (continuum mechanics, image analysis, signal processing and system identification), which was developed at The University of Auckland. The CMISS can be used to perform finite element, boundary element or finite difference analyses. It has a computational back end to perform the analyses and a graphical 


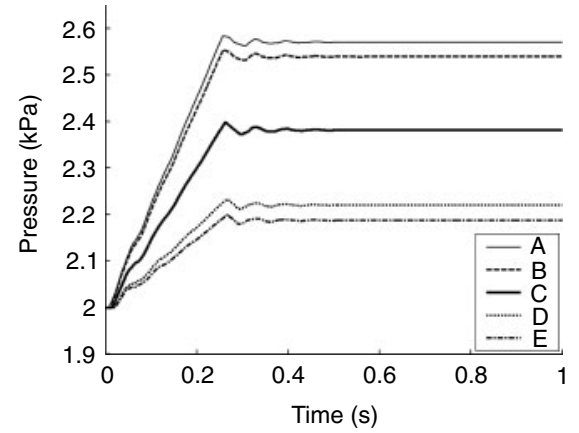

(a)

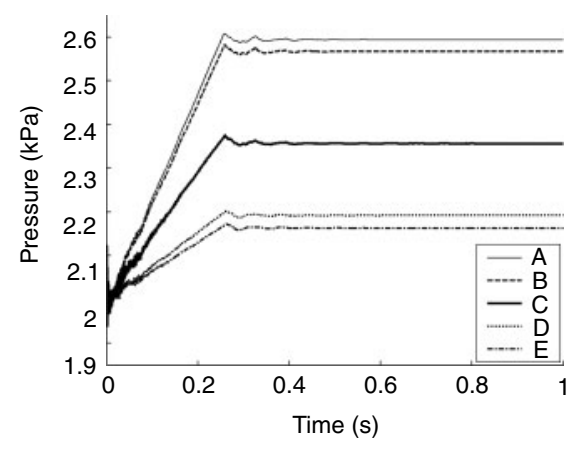

(d)

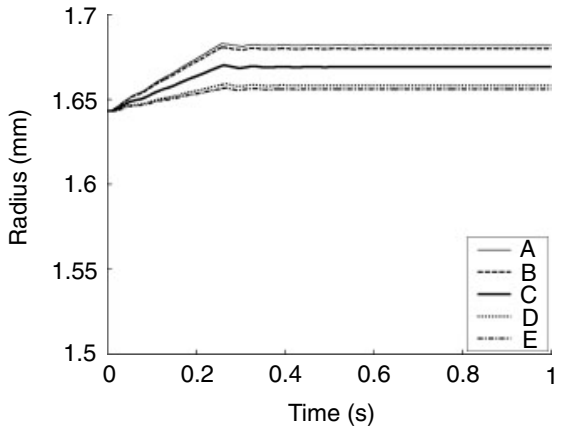

(b)

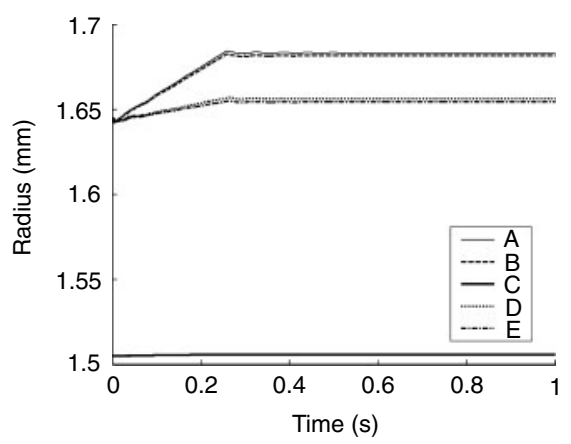

(e)

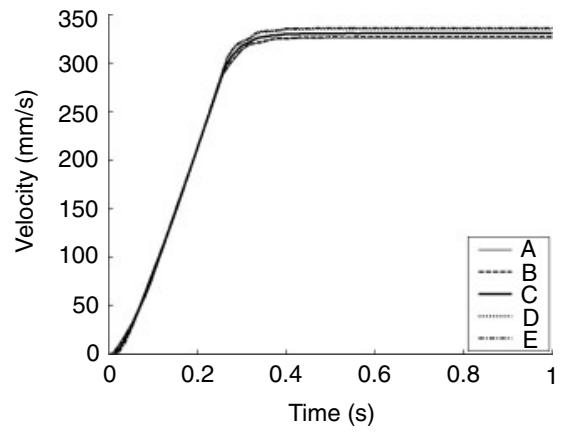

(c)

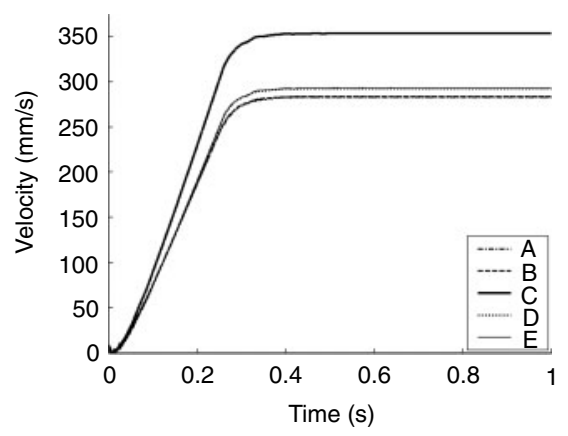

(f)

Figure 3 Time versus varying pressure, radius and flow velocity at various points (A-E) along the vessel segment 2 in the unstented ((a)-(c)) and stented ((d)-(f)) cases with linear increase in inlet pressure (Simulation I). See Figure 1 for the positions of these points in the network. Note that the radius at point $\mathrm{C}$ in the middle of the stented vessel is just over $1.5 \mathrm{~mm}$ (the radius of the stent).

front end allowing the user to view the results in three dimensions. From our previous study, we found that a time step of $2 \mathrm{e}-06 \mathrm{~s}$, when using double precision arithmetic, provides numerical stability, accuracy and no accumulation of numerical errors. Applying this time step, each simulation took approximately 25 minutes to run to a final simulation time of $1.0 \mathrm{~s}$.

\section{Simulation I}

Figure 3 presents time versus varying pressure, vessel radius and flow velocity results at different points along vessel segment 2 in the unstented and stented cases. The positions of these points on the network are shown in Figure 1. These points are significant for the following reasons: point A lies before the start of the region where the effects of the stent are felt (where the material properties of the vessel start changing), $\mathrm{B}$ lies at the start of the region where the effects of the stent are felt, $C$ lies exactly in the middle of the stented region where the vessel is at its stiffest, $D$ is the point corresponding to $\mathrm{B}$ downstream of the stent and $\mathrm{E}$ is the point corresponding to A downstream of the stent (i.e. after the material properties return to their unstented values).

The pressure plots in Figure 3 are similar to but not exactly the same as each other in the unstented and stented cases. In the stented case, the pressures at the upstream points are slightly higher and pressures at the downstream points are slightly lower than the corresponding values in the unstented case. Similarly, the radii upstream and downstream of the vessel are only slightly different in the stented and unstented cases. However, as expected, the radius in the middle of the stented region (point $\mathrm{C}$ ) hardly rises above $1.5 \mathrm{~mm}$ (the radius of the stent). Also, as expected, the velocity in the middle of the stent is much higher than the upstream or downstream velocities. The velocities upstream and downstream of the stent are lower in the stented case than in the unstented case.

Figure 4 shows that pressure along vessel segments 1, 2 and 4 at various times in the unstented and stented cases. No difference can be observed in the pressures for segment 1 . The greatest difference between the unstented and stented cases occurs in segment 2 where the stent is placed. In the transition zone to the stented region, the pressure dips at both the proximal and distal parts of the vessel. This introduces a short region where there is a negative pressure gradient. However, as can be seen in Figure 5(e), the blood still flows downstream due to the momentum already present in the flow. In segment 4 , there is a slight decrease in pressure in the stented case compared with the unstented case.

Figure 5 shows radius, velocity and flow along vessel segment 2 at various times in the unstented and stented cases. The radius in the stented region stays close to $1.5 \mathrm{~mm}$ 


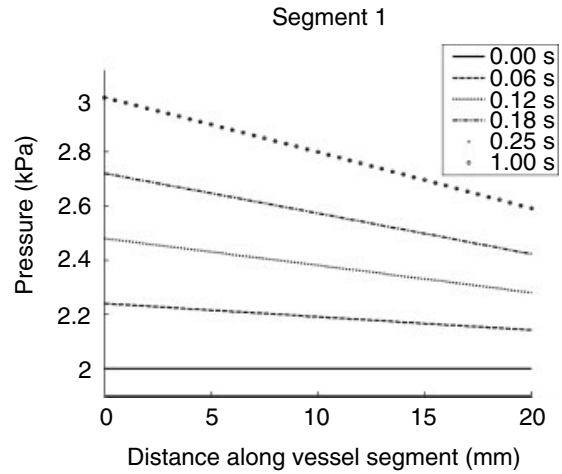

(a)

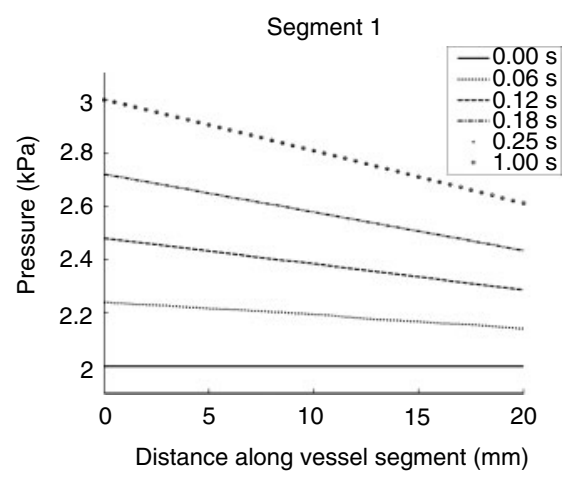

(d)

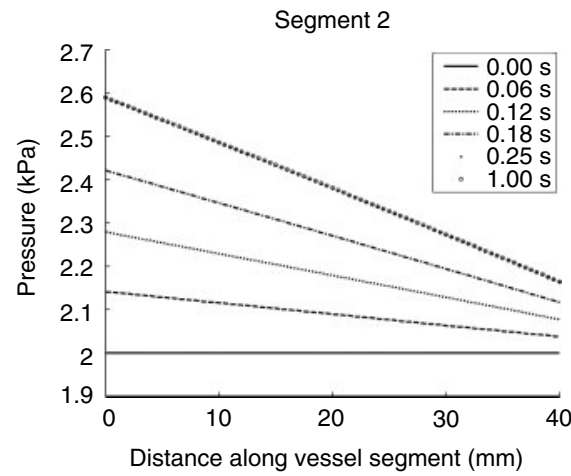

(b)

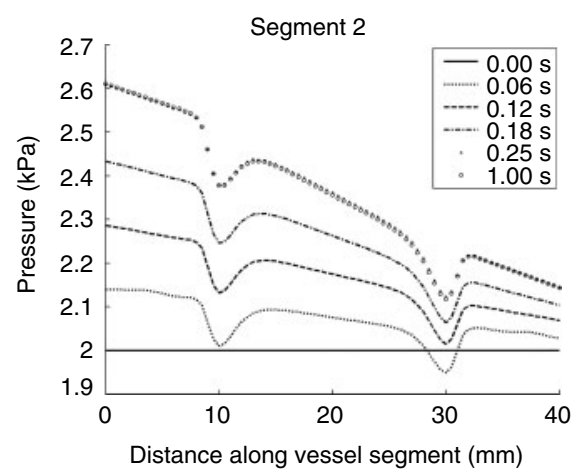

(e)

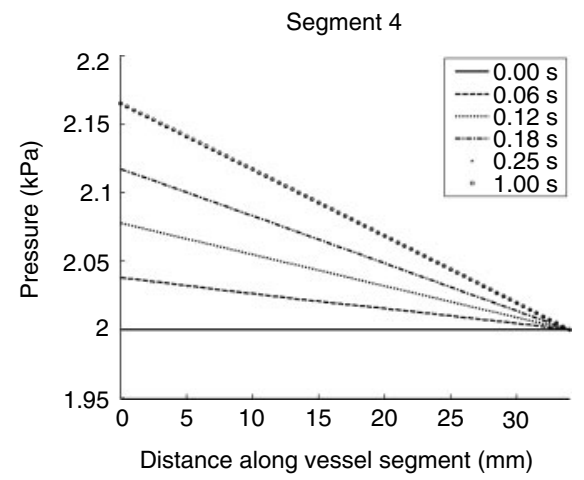

(c)

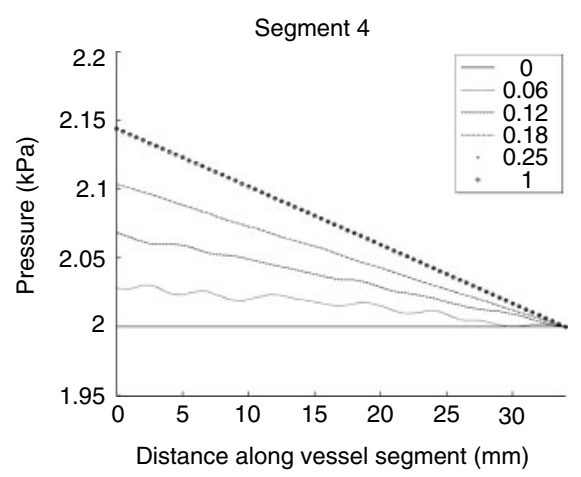

(f)

Figure 4 Pressure along different vessel segments at the times shown in the legends of the plots in the unstented ((a)-(c)) and stented ((d)-(f)) cases. The results for $0.25 \mathrm{~s}$ and $1.0 \mathrm{~s}$ are very similar to each other and appear together as the top line in all the plots. See Figure 1 for the positions of the vessel segments in the network.

as noted previously. The flow velocity along the unstented vessel is reasonably constant compared with the stented case. In the stented case, the velocity in the stented region of the vessel is greater than in the unstented regions. As the pressure increases over time, this difference in velocity also increases. The figure also shows that the flow rate in the unstented case is higher than in the stented case.

By $1.0 \mathrm{~s}$, the system essentially reaches a steady state. The radius along vessel segment 2 in the stented case at $1.0 \mathrm{~s}$ (from the finite difference solution) is used to compute the corresponding vessel area that is plotted against distance along the vessel in Figure 6. Also shown in the same figure is the solution of the steady-state ODE (16). Equation (16) was solved using a constant flow rate $(Q)$ of $2520 \mathrm{~mm}^{3} / \mathrm{s}$ and a vessel area $\left(S_{\mathrm{i}}\right)$ of $8.88 \mathrm{~mm}^{2}$ (at the start of the region that is modified by the stent, i.e. at $x=x_{\mathrm{s}}$ ). This steady-state flow rate and vessel cross-sectional area at $x_{\mathrm{s}}$ was obtained from the finite difference solution. Figure 6 also shows the pressure along the segment at $1.0 \mathrm{~s}$ from the finite difference and the steady-state ODE solutions. The steady-state ODE pressure was computed from the steady-state ODE area using (11).

\section{Simulation II}

Figure 7 presents time versus varying pressure, vessel radius and flow velocity results at different points along vessel segment 2 in the unstented and stented cases for sinusoidal inlet pressure variation. The positions of these points on the network are shown in Figure 1. As with the previous set of simulations (Simulation I: linear increase in inlet pressure), the pressure plots in Figure 7 are similar to but not exactly the same as each other in the unstented and stented cases. Similarly, the radii upstream and downstream of the vessel are only slightly different in the stented and unstented cases. The radius in the middle of the stented region (point $\mathrm{C}$ ) is only slightly higher than $1.5 \mathrm{~mm}$ (the radius of the stent). Also, as expected, the differences in velocity between the unstented and stented cases are as described for Simulation I.

Figure 8 shows pressure, radius and flow velocity along vessel segment 2 at various times in the unstented and stented cases. The trends in pressures, radii and flow velocities along the vessel described for Simulation I apply to this set of simulations as well. The pressure in the transition zone in the stented case shows the highest change from the unstented case.

\section{DISCUSSION}

The placement of a stent in an artery leads to a region of increased stiffness where the stent is placed. Because of this increase in stiffness, the flow is changed considerably 


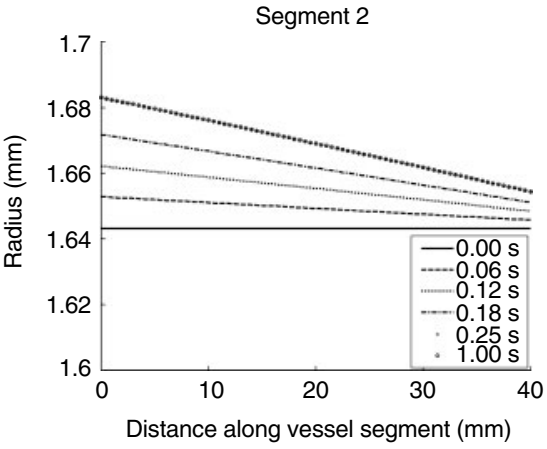

(a)

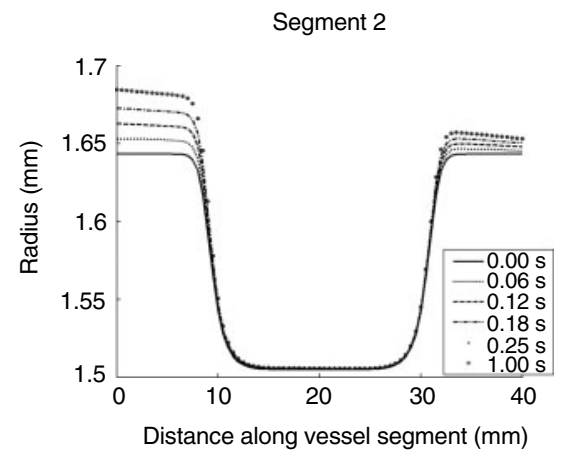

(d)

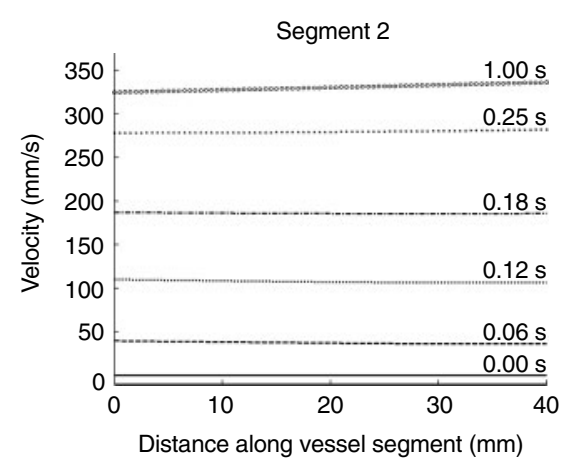

(b)

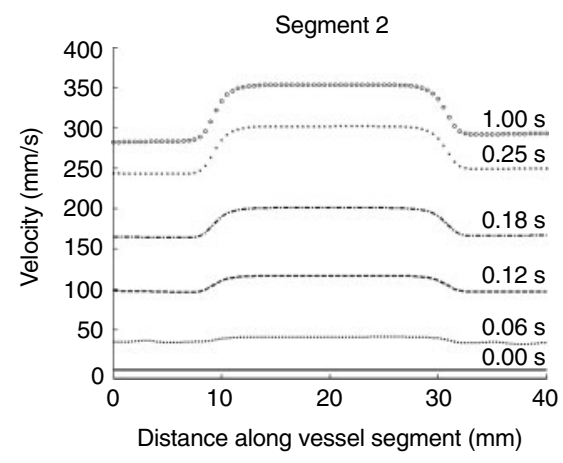

(e)

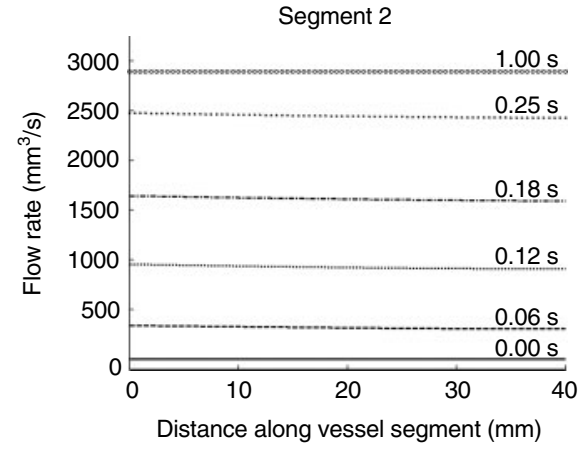

(c)

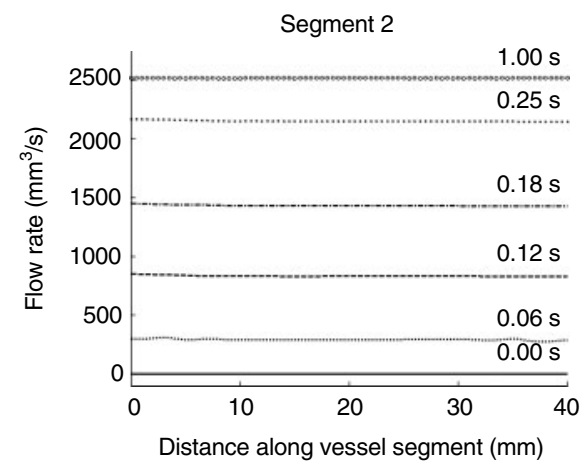

(f)

Figure 5 Radius, flow velocity and flow rate along vessel segment 2 at the times shown in the legends of the plots in the unstented ((a)-(c)) and stented ((d)-(f)) cases. The radius results for $0.25 \mathrm{~s}$ and $1.0 \mathrm{~s}$ are very similar to each other and appear together as the top line in plots (a) and (d). See Figure 1 for the positions of the vessel segment 2 in the network.

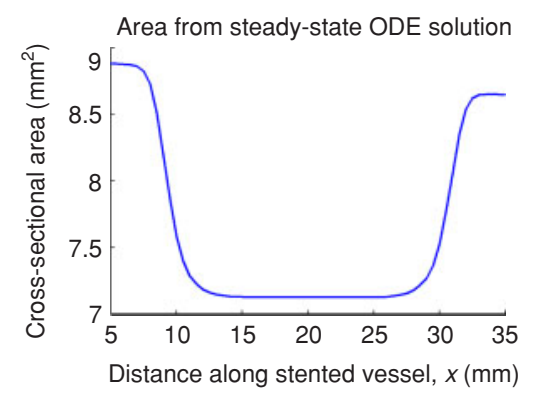

(a)

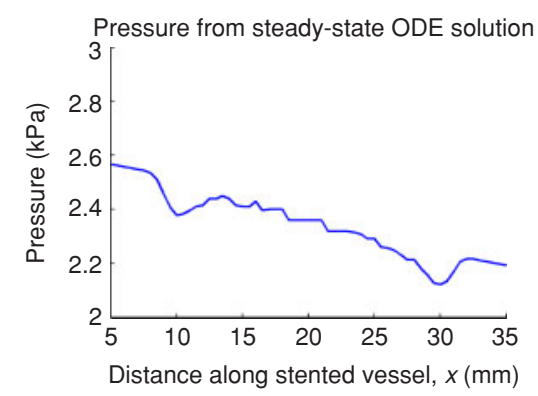

(c)

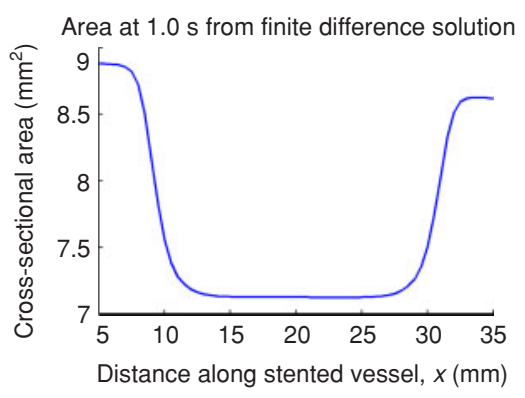

(b)

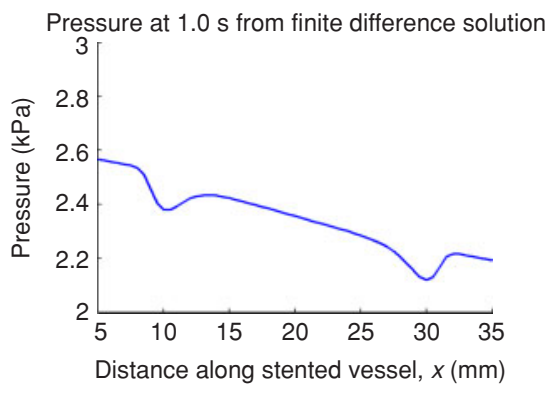

(d)

Figure 6 Area and pressure from steady-state ODE solution ((a) and (c)) and from finite difference solution at a simulation time of $1.0 \mathrm{~s}((\mathrm{~b})$ and $(\mathrm{d}))$. The steady-state ODE solution does not appear as smooth as the finite difference solution due to numerical errors introduced by the ODE solver. 


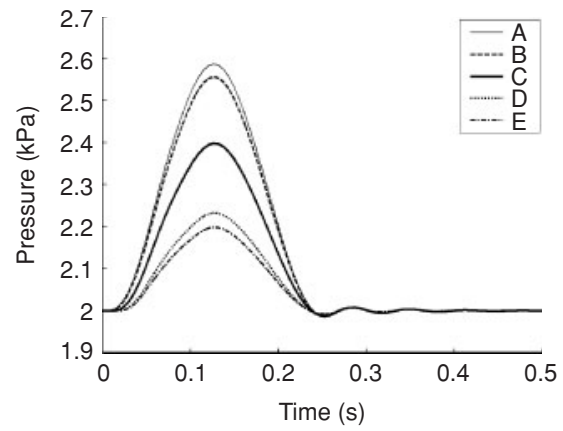

(a)

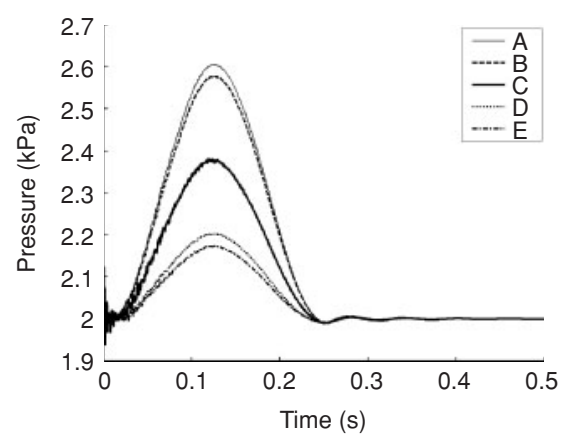

(d)

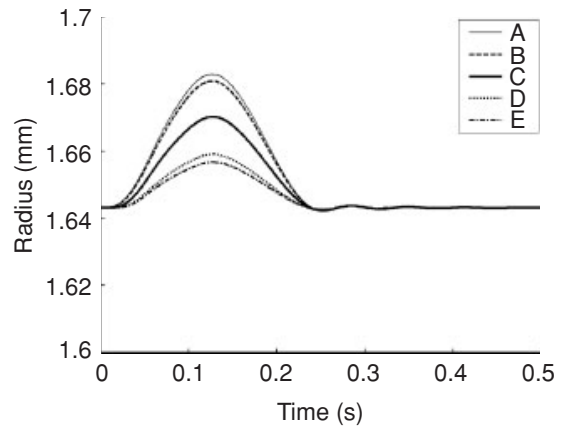

(b)

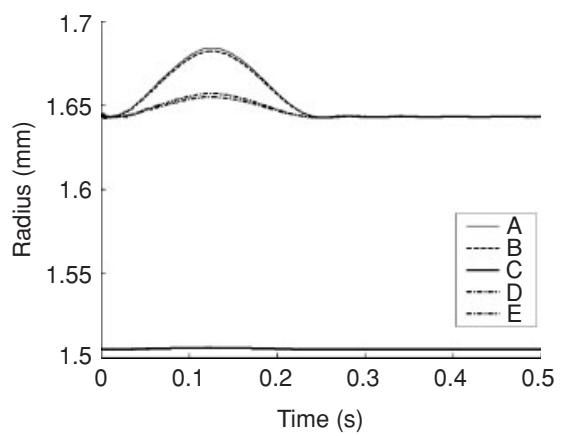

(e)

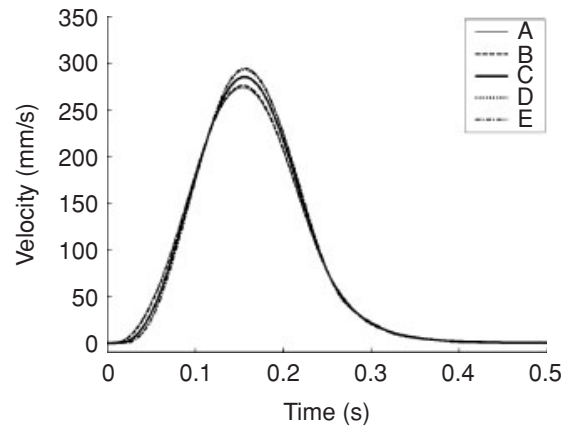

(c)

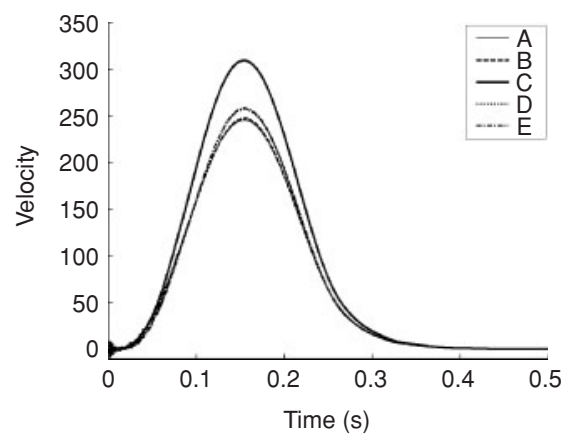

(f)

Figure 7 Time versus varying pressure, radius and flow velocity at various points (A-E) along the vessel segment 2 in the unstented ((a)-(c)) and stented ((d)-(f)) cases with sinusoidal inlet pressure variation (Simulation II). See Figure 1 for the positions of these points in the network. Note that the radius at point $\mathrm{C}$ in the middle of the stented vessel is just over $1.5 \mathrm{~mm}$ (the radius of the stent).

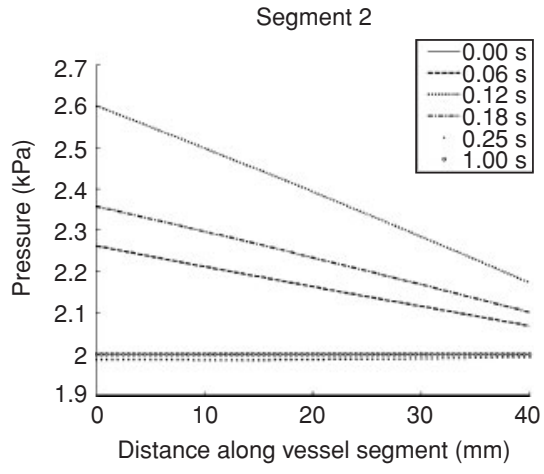

(a)

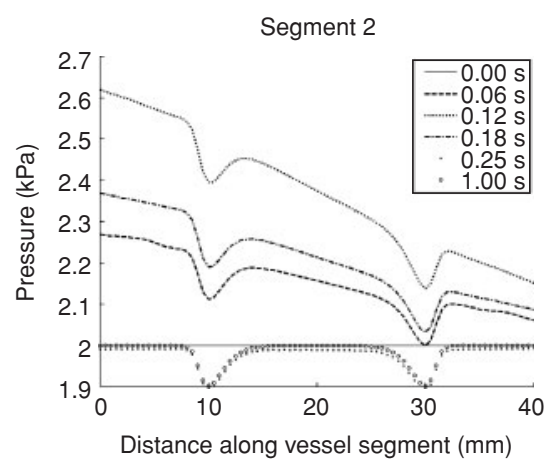

(d)

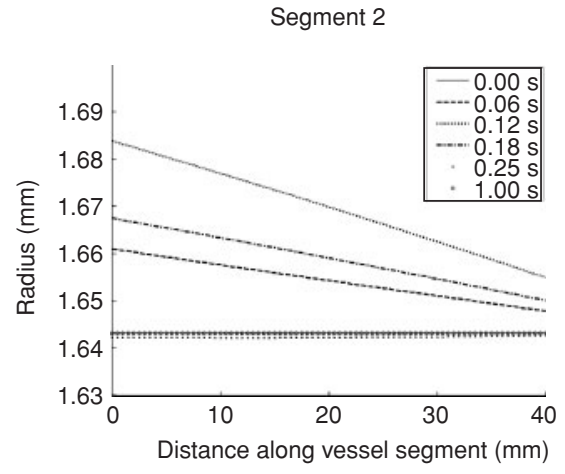

(b)

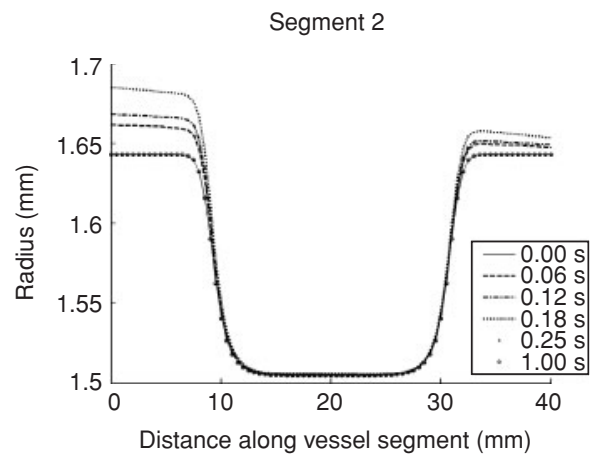

(e)
Segment 2

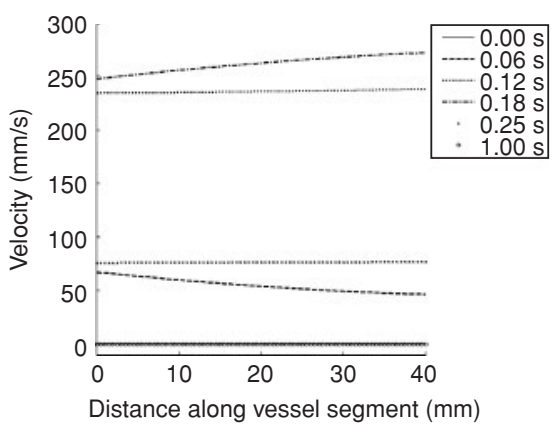

(c)

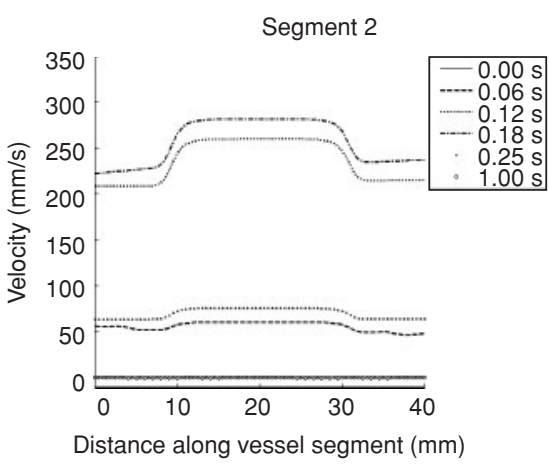

(f)

Figure 8 Pressure, radius and flow velocity along vessel segment 2 at the times shown in the legends of the plots in the unstented ((a)-(c)) and stented ((d)-(f)) cases. See Figure 1 for the positions of the vessel segment 2 in the network. 
within the stented region and at the transition from the unstented to stented regions. This study makes two contributions - firstly, we extend a computationally efficient methodology to solve for flow in a stented coronary artery network and, secondly, using this methodology, we investigate flow in a coronary artery network with and without the stent in place.

The strength of the methodology presented in this article lies in the fact that it can be implemented in a full coronary artery mesh relatively easily following the procedure used by Smith et al. (2002). The framework for embedding this full coronary mesh in a model of the heart is also in place (Smith et al. 2000, 2005). The flow problem can be combined with the deformation of the myocardium of the heart to produce more realistic simulations of blood flow in stented and unstented networks. Another advantage of the methodology presented in this study is its ability to simulate the effects of more than one stent at various places in the network.

The main limitation of this methodology is its onedimensional nature and the lack of localised flow information that can only be obtained with higher dimensional models. Local pressure gradients, changes in velocity and wall shear stress can be computed accurately with three-dimensional models of the stented region. However, when considering the effects of flow changes in large networks of vessels such as the coronary network, full threedimensional models are computationally prohibitive while one-dimensional models provide the required computational efficiency.

We have applied two relatively simple pressure boundary conditions in this study. It is important to acknowledge that the actual pressure gradients in the coronary vasculature can be determined by intermyocardial, ventricular and transmission of downstream coronary pressure. Thus, it is impossible to say whether a constant or sinusoidal boundary condition is more appropriate. However, we do propose that the two boundary conditions applied provide an initial basis from which to understand the dynamics of the system.

The results of the investigation of flow in a coronary artery network with and without a stent in place allow us to draw conclusions regarding restenosis and arterial remodelling. As mentioned previously, a significant problem arises due to restenosis of stented regions and remodelling of other arterial segments. Restenosis is thought to be the result of abnormal flow conditions affecting the endothelial cell (innermost) layer of the blood vessel. Vessel wall shear stress distribution and flow direction are known to affect endothelial cells (Davies 1995; Mates 1995; Kataoka et al. 1998; Yamamoto et al. 2003).

Wall shear stress is dependent on local velocity gradients, which in turn are dependent on the pressure gradients along the vessel. Rapidly changing pressure gradients such as those occurring in the transition zones between the stented and unstented regions of a vessel (Figures 4 and 8 ) could indicate local regions of negative velocity, although the average velocity is positive (Figures 5 and 8). The rapidly changing pressure gradients indicate the possibility of a large difference in the mechanical environment of endothelial cells at the entrance and exit of stented regions in vessels. As recently reviewed by Boisseau (2005), this has significant implications for gene regulation, vulnerability to endothelial cell hypoxia, accumulation of white cells and a number of other haemorheological disorders. Furthermore, these changes in local flow velocity can adversely affect the endothelial cell layer and hence cause restenosis.

Wentzel et al. (2000) found significant changes in wall shear stresses at the entrance and exit areas of the stent, and concluded that the changes could be related to in-stent restenosis. Moore and Berry (2002) also confirmed that the greatest effects are likely to be felt at the entrance and exit areas of the stents. Rachev et al. (2000) mentioned experimental studies that have found arterial lumen decreasing in the region just outside the stent due to wall remodelling. Their results showed the remodelling effects as a result of axial and circumferential stress concentration in the immediate vicinity of the stent.

A very important factor in any model regarding the effects of the stent is the set of material properties or the particular form of the pressure-radius relationship assigned to the region in question. The model presented in this study can be used to assess the effects of different material property assumptions on coronary blood flow in detailed representations of coronary vasculature.

\section{REFERENCES}

Barragan P, Rieu R, Garitey V, et al. 2000. Elastic recoil of coronary stents: a comparative analysis. Catheter Cardiovasc Interv, 50:112-9.

Berry JL, Santamarina A, Moore JE Jr, et al. 2000. Experimental and computational flow evaluation of coronary stents. Ann Biomed Eng, 28:386-98.

Boisseau MR. 2005. Roles of mechanical blood forces in vascular diseases. A clinical overview. Clin Hemorheol Microcirc, 33(3):201-7.

Capozzolo C, Piscione F, De Luca G, et al. 2001. Direct coronary stenting: effect of coronary blood flow, immediate and late clinical results. Catheter Cardiovasc Interv, 53:464-73.

Carmines DV, McElhaney JH, Stack R. 1991. A piece-wise non-linear elastic stress expression of human and pig coronary arteries tested in vitro. 7 Biomech, 24:899-906.

Davies PF. 1995. Flow-mediated endothelial mechanotransduction. Physiol Rev, 75:519-60.

Defily D, Kuo L, Davis M, et al. 1993. Segmental Distribution and Control of Coronary Microvascular Resistance. New York: Springer-Verlag, p. 270-82.

Dormand JR, Prince PJ. 1980. A family of embedded Runge-Kutta formulae. 7 Comp Appl Math, 6:19-26.

Etave F, Finet G, Biovin M, et al. 2001. Mechanical properties of coronary stents determined by using finite element analysis. $\mathcal{F}$ Biomech, 34:1065-75

Formaggia L, Lamponi D, Quarteroni A. 2003. Dimensional models for blood flow in arteries. 7 Eng Math, 47(3-4): 251-76. 
Hsieh I, Chien C, Chang H, et al. 2001. Acute and long-term outcomes of stenting in coronary vessel $>3.0 \mathrm{~mm}$, and $<2.5 \mathrm{~mm}$. Catheter Cardiovasc Interv, 53:314-22.

Jowett NI, Thompson DR. 2003. Comprehensive Coronary Care. 3rd ed. Edinburgh: Bailliere Tindall.

Kataoka N, Ujita S, Sato M. 1998. Effect of flow direction on the morphological responses of cultured bovine aortic endothelial cells. Med Biol Eng Comput, 36:122-8.

LaDisa JF Jr, Guler I, Olson LE, et al. 2003. Three-dimensional computational fluid dynamics modeling of alterations in coronary wall shear stress produced by stent implantation. Ann Biomed Eng, 31:972-80.

Mates RE. 1995. Arterial macrocirculatory hemodynamics. In Bronzino JD, eds. The Biomedical Engineering Handbook. Salem: CRC Press, p. 454-62.

Migliavacca F, Petrini L, Colombo M, et al. 2002. Mechanical behavior of coronary stents investigated through the finite element method. 7 Biomech, 35:803-11.

Moore JE Jr, Berry JL. 2002. Fluid and solid mechanical implications of vascular stenting. Ann Biomed Eng, 30:498-508.

Rachev A, Manoach E, Berry J, et al. 2000. A model of stressinduced geometrical remodeling of vessel segments adjacent to stents and artery/graft anastomoses. 7 Theor Biol, 206:429-43.

Rolland PH, Charifi A-B, Verrier C, et al. 1999. Hemodynamics and wall mechanics after stent placement in swine iliac arteries: comparative results from six stent designs. Radiology, 213:229-46.

Sherwin S, Franke V, Peiro J, et al. 2003. One-dimensional modelling of a vascular network in space-time variables. $\mathcal{F}$ Eng Math, 47(3-4):217-50.
Smith NP, Pullan AJ, Hunter PJ. 2000. Generation of an anatomically based geometric coronary model. Ann Biomed Eng, 28:14-25.

Smith NP, Pullan AJ, Hunter PJ. 2002. An anatomically based model of transient coronary blood flow in the heart. SIAM 7 Appl Math, 62:990-1018.

Smith NP, Stevens C, Hunter PJ. 2005. Computational modeling of ventricular mechanics and energetics. Appl Mech Rev, 58(2):77-90.

Tan LB, Webb DC, Kormi K, et al. 2001. A method for investigation the mechanical properties of intracoronary stents using finite element numerical simulation. Int 7 Cardiol, 78:51-67.

Tortoriello A, Pedrizzetti G. 2004. Flow-tissue interaction with compliance mismatch in a model stented artery. $\mathcal{F}$ Biomech, 37(1):1-11.

Wentzel JJ, Whelan DM, van der Giessen WJ, et al. 2000. Coronary stent implantation changes $3-\mathrm{D}$ vessel geometry and 3-D shear stress distribution. 7 Biomech, 33:1287-95.

Yamamoto K, Sokabe T, Ohura N, et al. 2003. Endogenously released ATP mediates shear stress-induced $\mathrm{Ca}^{2+}$ influx into pulmonary artery endothelial cells. Am $\mathcal{F}$ Physiol-Heart Circ Physiol, 285:H793-H803.

Yock CA, Boothroyd DB, Owens DK, et al. 2003. Cost-effectiveness of bypass surgery versus stenting in patients with multivessel coronary artery disease. $\mathrm{Am} \mathcal{F} \mathrm{Med}$, 115:382-9.

Zhu H, Warner JJ, Gehrig TR, et al. 2003. Comparison of coronary artery dynamics pre- and post-stenting. $\mathcal{F}$ Biomech, 36:68997. 

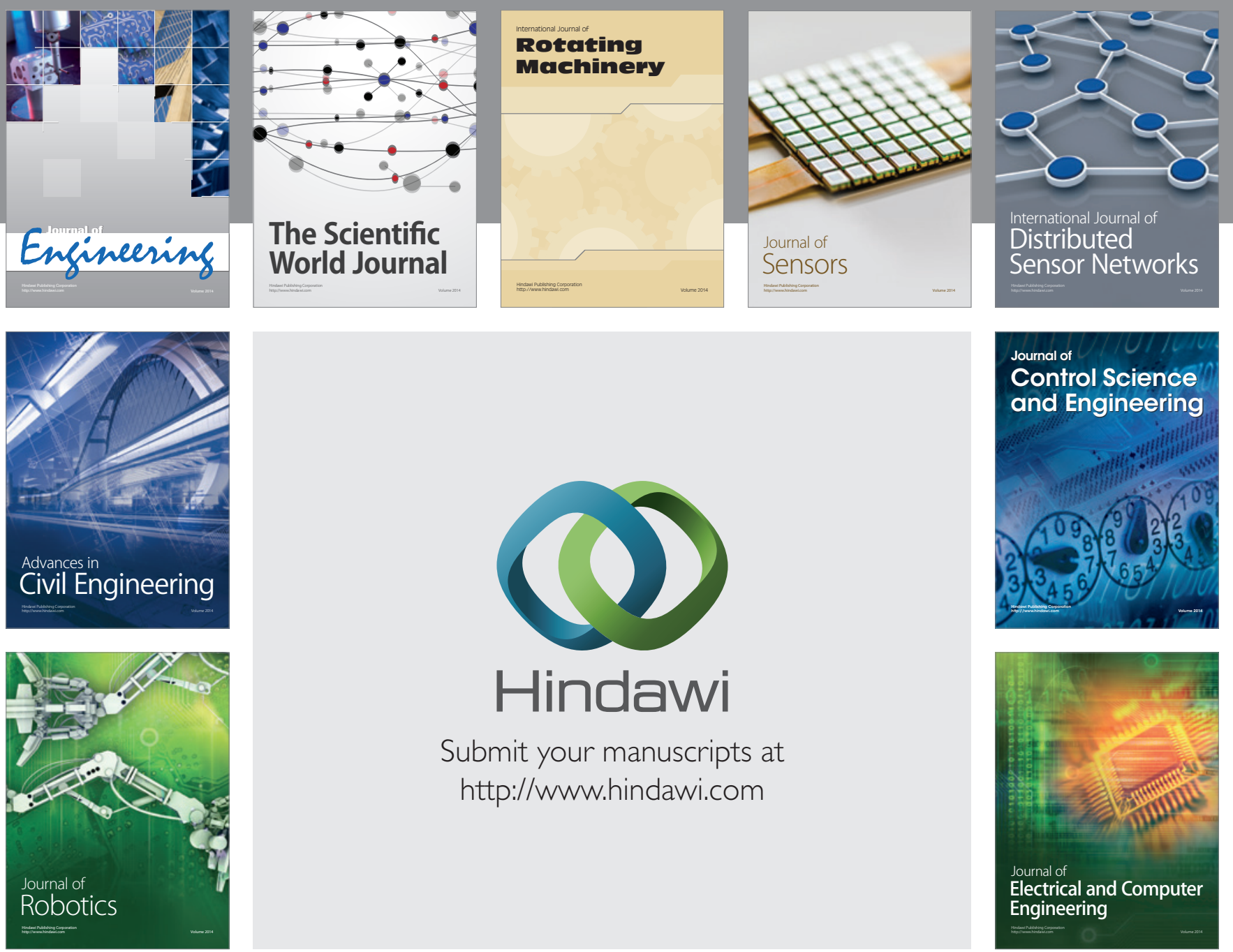

Submit your manuscripts at

http://www.hindawi.com
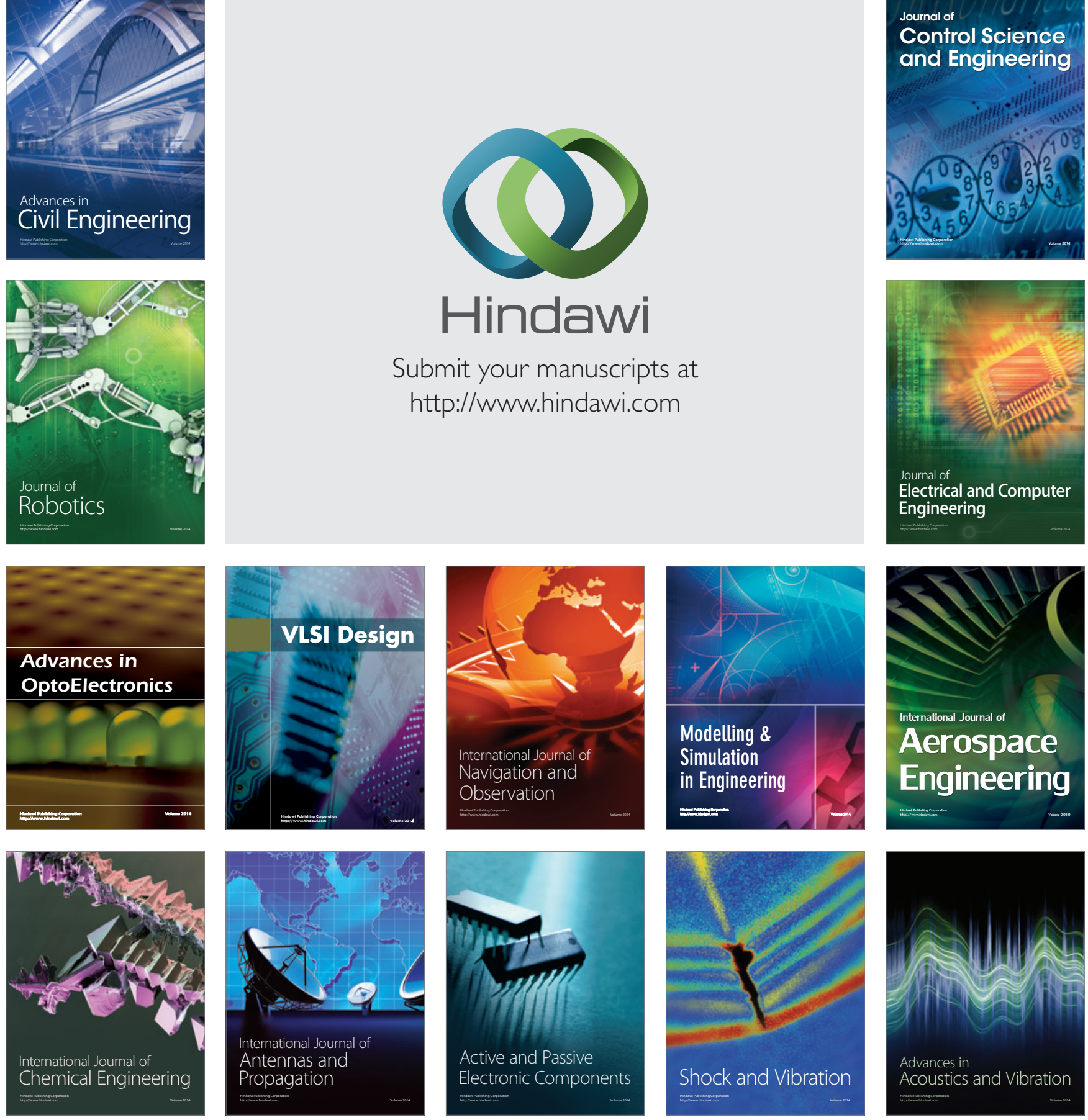